\title{
Elevated neutrophil-to-lymphocyte ratio is associated with nutritional impairment, immune suppression, resistance to $S-1$ plus cisplatin, and poor prognosis in patients with stage IV gastric cancer
}

\author{
KENJI GONDA $^{1,2}$, MASAHIKO SHIBATA ${ }^{2,3}$, YU SATO ${ }^{2,4}$, MARIA WASHIO ${ }^{5,6}$, HIROYUKI TAKESHITA ${ }^{5,7}$, \\ HIROFUMI SHIGETA $^{5,8}$, MICHIKAZU OGURA ${ }^{5,9}$, SHINICHI OKA ${ }^{5,10}$ and SHINICH SAKURAMOTO 5

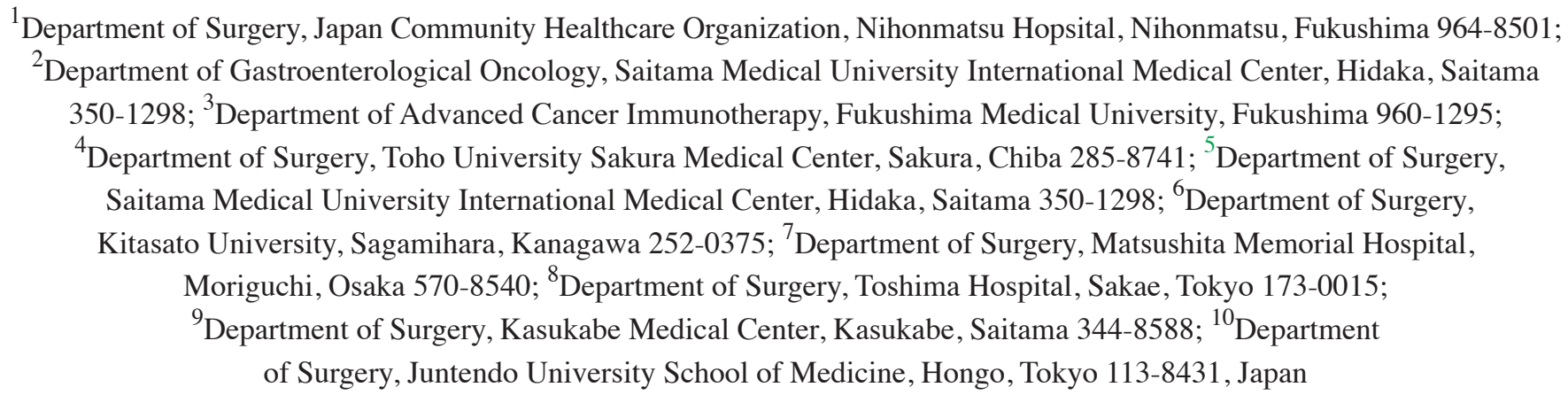

Received April 1, 2017; Accepted September 28, 2017

DOI: $10.3892 / \mathrm{mco} .2017 .1438$

\begin{abstract}
Gastric cancer continues to be a major cause of morbidity and mortality worldwide. Recently, there has been a growing interest in the host inflammatory response and there is increasing evidence that the neutrophil to lymphocyte ratio (NLR), which is a useful marker of systemic inflammation, can be an effective prognostic indicator in various types of malignant diseases. A total of 110 patients with stage IV gastric cancer who received chemotherapy of S-1 plus cisplatin were enrolled in this study. Eleven patients did not complete four cycles of the chemotherapy. The patients were divided into two groups with 3.0 of NLR. The percentage of patients with a partial response to chemotherapy was significantly higher in the group of patients with a lower NLR $(<3)(19.1$ vs. $38.5 \%$, high vs. low NLR group, respectively; $\mathrm{P}<0.05)$. The percentage of patients with progressive disease was higher in the high vs. low NLR group (57.4 vs. $25.0 \%$, respectively; $\mathrm{P}<0.05$ ). NLR levels were significantly inversely correlated with serum
\end{abstract}

Correspondence to: Dr Kenji Gonda, Department of Surgery, Japan Community Healthcare Organization, Nihonmatsu Hospital, 1-553 Naritamachi, Nihonmatsu, Fukushima 964-8501, Japan E-mail: gonken@lime.plala.or.jp

Key words: neutrophil-to-lymphocyte ratio, immune suppression, Gastric cancer, inflammation, TS1+cisplatin, malnutrition, overall survival rate levels of prealbumin $(\mathrm{P}<0.01)$ and retinol binding protein $(\mathrm{P}<0.05)$. NLR levels were also significantly correlated with c-reactive protein levels $(\mathrm{P}<0.05)$, white blood cell count $(\mathrm{P}<0.05)$ and inversely with the stimulation index (a marker of cell-mediated immune function; $\mathrm{P}<0.05)$. Overall survival was significantly longer in patients with a lower NLR $(\leq 3.0)$ than in those with a higher NLR (>3.0). The present study demonstrated that the NLR is a useful marker for resistance to chemotherapy, malnutrition, systemic inflammation and immune suppression. Moreover, the NLR was demonstrated to be a strong prognostic indicator in these patients.

\section{Introduction}

Gastric cancer continues to be a major cause of morbidity and mortality worldwide (1). According to the International Agency for Research on Cancer (IARC), one million new cases of gastric cancer are diagnosed each year, and gastric cancer causes over 700,000 deaths worldwide each year (2). Despite an improvement in survival over recent years due to the development of better endoscopic and imaging techniques, surgical procedures and skills, and oncological treatments, its prognosis is still unfavorable.

Recently, there has been a growing interest in the host inflammatory response to tumors, and the systemic inflammatory response has been shown to reflect the promotion of angiogenesis, DNA damage, and tumor invasion through the over-production of cytokines (3-5). Based on these findings, a number of inflammation-based prognostic markers such as the Glasgow Prognostic Score (GPS) and the platelet to lymphocyte ratio (PLR) have been studied $(6,7)$. In addition, 
there is increasing evidence that the neutrophil to lymphocyte ratio (NLR) can be an effective prognostic indicator in various types of malignant diseases (8-18).

In cancer patients, lymphopenia reflects an impaired cell-mediated immunity, while neutrophilia is acknowledged as a response to systemic inflammation. The NLR has been suggested to be a marker for general immune responses to various stress stimuli. The NLR has also been reported to correlate with the severity of clinical progress in severely ill patients in the intensive care unit; emerging evidence has shown that the NLR has a prognostic value in patients with solid tumors (19-21).

To the best of our knowledge, no studies of NLR regarding responses to chemotherapy against gastric cancer, or its correlation with patients' nutritional status have been published. In this study, we therefore evaluated the clinical utility of the NLR in patients with stage IV gastric cancer under pre-treatment conditions.

S-1 (TS-1, Taiho Pharmaceutical Co., Ltd) is an oral fluoropyrimidine agent that is designed to have strong anti-cancer activity and to have reduced gastrointestinal toxicity compared to other anti-cancer drugs. It consists of tegafur, 5-chloro-2, 4-dehydroxypyrimidine (an inhibitor of dihydropyrimidine dehydrogenase), and potassium oxonate dehydrogenase (an inhibitor of phosphoribosyl transferase) in a molar ratio of 1:0.4:1 (22). S-1 plus cisplatin has been reported have significant effectivity for unresectable or recurrent gastric cancer and is recognized as a standard therapy in Japan (23-26).

Cancer cachexia, another important problem in cancer treatment and care, is associated with nutritional impairment and immune suppression $(27,28)$. We previously reported that malnutrition or hypoalbuminemia shows a good correlation with immune suppression, as well as with systemic inflammation (29-33). Malnutrition has also been reported to show a good correlation with the suppression of cell-mediated immunity. Systemic inflammation may underlie these important conditions that are prominent in patients with advanced cancer. Relationships between the NLR and nutritional status or immune function were therefore also assessed in the present study.

\section{Patients and methods}

Patients. We enrolled 110 patients with stage IV gastric cancer who received chemotherapy of S-1 plus cisplatin between May 2013 and June 2015 at Saitama Medical University International Medical Center. Details of these patients are listed in Table I. The study was approved by the institutional review board of Saitama Medical University International Medical Center (14-107). All patients provided written informed consent. This retrospective study was performed on all consecutive patients referred for chemotherapy treatment for gastric cancer after invasive diagnostic and staging workup at our unit. Data were extracted from electronic medical files. Patients entered in this study were aged 35-80 (66.2 median) years and had an Eastern Cooperative Study Group (ECOG) performance status (PS) of 0 or 1 .

Markers for nutrition, immune reaction and prognosis. The NLR was defined as follows: NLR=peripheral neutrophil
Table I. Study participant characteristics.

\begin{tabular}{lc}
\hline Characteristics & Values \\
\hline Sex (male:female) & $56: 54$ \\
Age (years) & $66.2(35-80)$ \\
Primary tumor stage (T1-3:T4) & $24: 86$ \\
Nodal involvement (N0:N1-3) & $27: 83$ \\
Distant metastasis (M0:M1) & $22: 88$ \\
Serum levels of CEA $(<5.0:>5.0)$ & $36: 74$
\end{tabular}

Groups were balanced on sex, age. But, participants were fairly balanced on tumor node metastasis classification. CEA, carcinoembryonic antigen.

Table II. Summary of response rate after chemotherapy.

\begin{tabular}{ccccc}
\hline NLR & CR & PR $^{\mathrm{a}}$ & $\mathrm{SD}$ & $\mathrm{PD}^{\mathrm{a}}$ \\
\hline$>3.0$ & 0 & 19.1 & 23.4 & 57.4 \\
$<3.0$ & 0.2 & 38.5 & 34.6 & 25 \\
\hline
\end{tabular}

${ }^{\mathrm{a}} \mathrm{P}<0.05$. The preoperative patients were separated into 2 groups based on whether NLR were $>$ or $<3.0$. The response rate of these 2 groups was then compared. In the PD patients, response rate with NLR $>3.0$ was significantly more than that with NLR $<3.0$. On the other hand, in the PR patients, response rate with NLR $>3.0$ was significantly less than that with $\mathrm{NLR}<3.0$. PR, partial response rate; $\mathrm{PD}$, progressive disease rate; $\mathrm{CR}$, complete response; $\mathrm{SD}$, stable disease.

count/peripheral lymphocyte count. Peripheral blood was drawn and was used for a PHA-lymphocyte-proliferation assay for measurement of the activity of cell-mediated immunity. The serum concentrations of prealbumin (PA) and retinol binding protein (RBP), which are both rapid turnover proteins (RTPs), were measured as nutritional parameters, using immunoturbidimetry and latex flocculation turbidimetry, respectively. The serum levels of c-reactive protein (CRP) were measured as an inflammatory indicator, using latex flocculation turbidimetry.

Chemotherapy with $S-1$ plus cisplatin. All of the patients received $80 \mathrm{mg} / \mathrm{m}^{2}$ oral S-1 in two $40 \mathrm{mg} / \mathrm{m}^{2}$ doses daily after meals on days $1-21$ and cisplatin $\left(60 \mathrm{mg} / \mathrm{m}^{2}\right)$ as an i.v. infusion on day 8 , repeated every 5 weeks. The dose of S-1 was assigned according to body surface area (BSA) as follows: $\mathrm{BSA}<1.25 \mathrm{~m}^{2}, 80 \mathrm{mg} /$ day; BSA 1.25 to $1.50 \mathrm{~m}^{2}, 100 \mathrm{mg} / \mathrm{day}$, and BSA $1.5 \mathrm{~m}^{2}$ or higher, $120 \mathrm{mg} /$ day. This combination chemotherapy was repeatedly administered for a total of 4 cycles and the responses were then evaluated according to the Response Evaluation Criteria in Solid Tumors (RECIST), version 1.0. The patients who responded to this therapy (with stable disease or partial remission) received the same chemotherapy regimen, and those who did not respond to this treatment (with progressive diseases) received ramucirumab combined with paclitaxel and/or irinotecan as second and third line chemotherapy. 

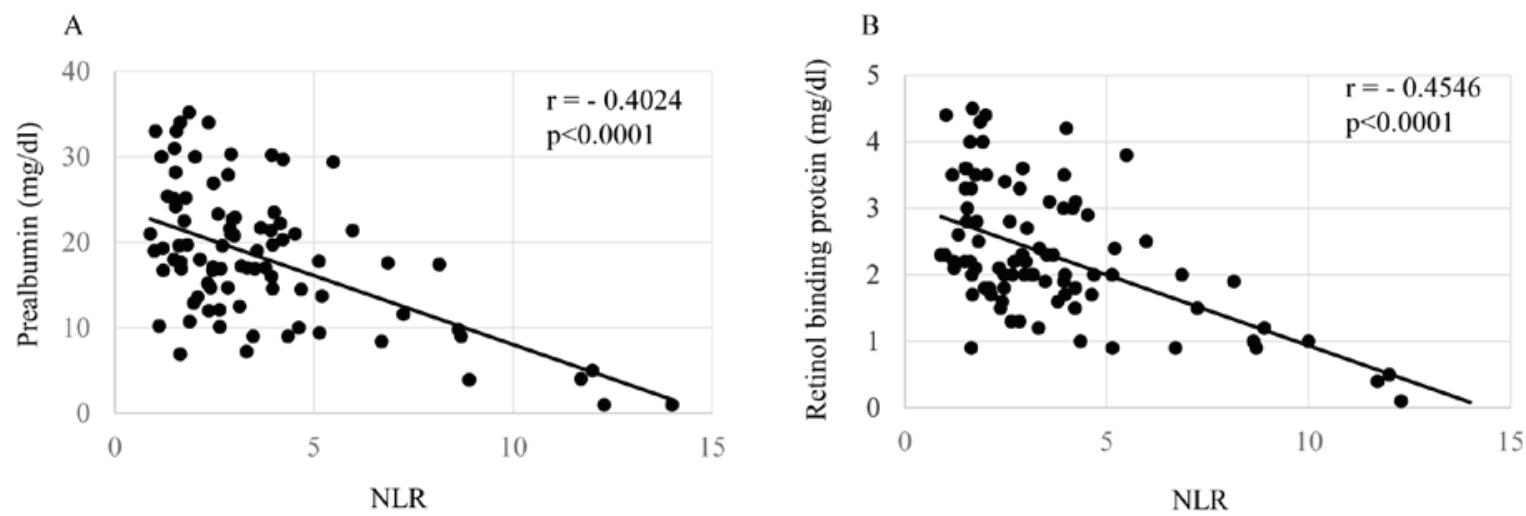

Figure 1. Nutrition and NLR. In preoperative gastric cancer patients, NLR were significantly negatively correlated with (A) prealbumin and (B) retinol binding protein. NLR, neutrophil/lymphocyte ration.
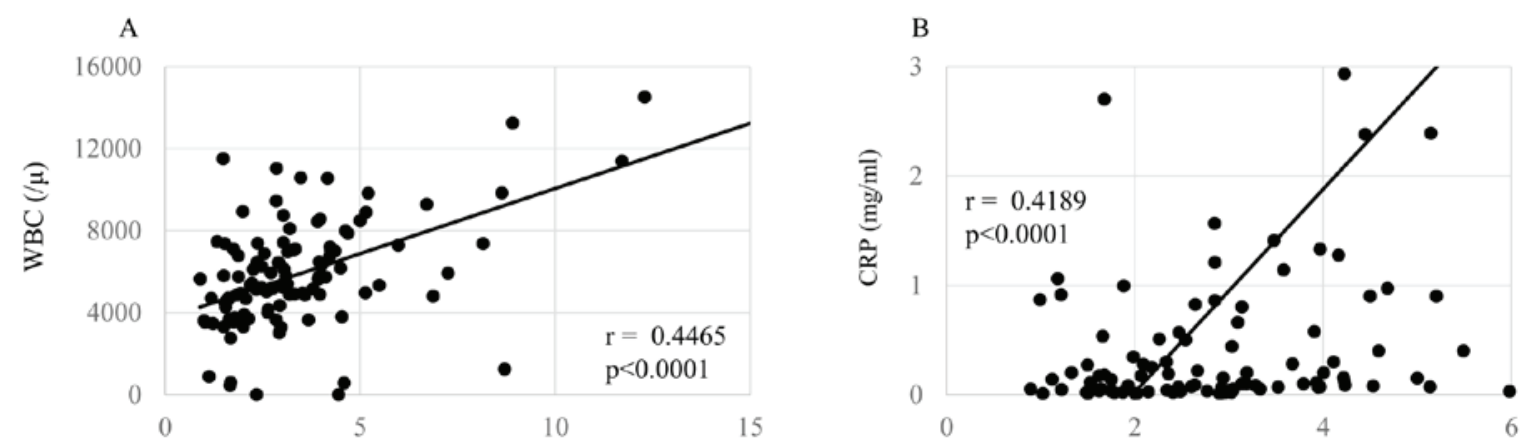

Figure 2. Inflammation and NLR. In preoperative gastric cancer patients, NLR were significantly positively correlated with (A) WBC and (B) CRP. NLR, neutrophil/lymphocyte ration; CRP, C-reactive protein.

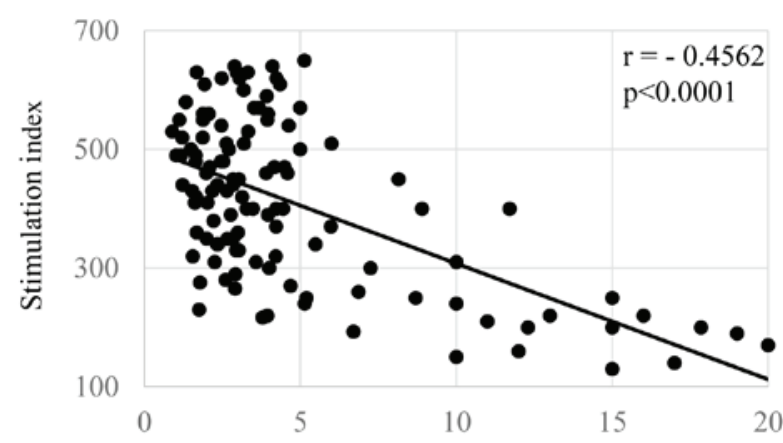

Figure 3. SI and NLR. In preoperative gastric cancer patients, NLR were significantly negatively correlated with SI. NLR, neutrophil/lymphocyte ration; SI, stimulation index.

Statistical analysis. SAS software version 9.2 (SAS Institute Inc, Cary, NC, USA) was used for statistical analysis. The significance of the correlations between parameters including the NLR was analyzed by the $\chi^{2}$ test and a t-test. Survival curves were estimated using the Kaplan-Meier method and the log-rank test was used to compare the survival curves. $\mathrm{P}=0.042$ was considered statistically significant. The patients were divided into 2 groups based on a cut-off NLR level of 3.0. The survival and the response to chemotherapy of the 2 groups were compared. Overall survival (OS) was defined as the time from the starting date of chemotherapy until death.

The results of Cox proportional hazards model analyzing factors associated with OS are shown in Table III.

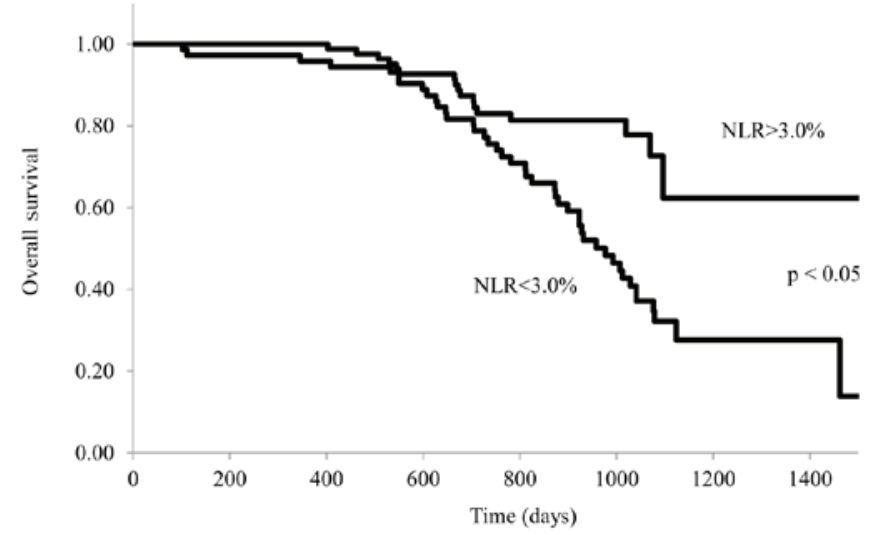

Figure 4. NLR of patients with stage IV gastric cancer and overall survival. Kaplan-Meier analysis for overall survival according to NLR. The patients were stratified into two groups: NLR $<3.0 \%$ and NLR $>3.0 \%$. Survival analysis was performed in 110 stage IV patients with follow-up.

\section{Results}

NLR and chemotherapy outcome. Among the 110 patients with Stage IV gastric cancer enrolled in the study, 11 patients did not complete 4 cycles of the S-1 plus cisplatin chemotherapy because of grade 3 gastrointestinal adverse effects and a decrease in PS. The responses to chemotherapy based on RECIST criteria of the high and low NLR patient groups are shown in Table II. The percentage of patients with a partial 
Table III. Cox proportional hazards model of overall survival in patients with gastric cancer with receiving chemotherapy

\begin{tabular}{|c|c|c|c|c|c|c|c|c|c|}
\hline \multirow[b]{2}{*}{ Marker } & \multirow[b]{2}{*}{ No. } & \multicolumn{4}{|c|}{ Univariable } & \multicolumn{4}{|c|}{ Multivariable } \\
\hline & & P-value & HR & $95 \% \mathrm{LB}$ & $95 \%$ UB & P-value & HR & $95 \%$ LB & $95 \% \mathrm{UB}$ \\
\hline \multicolumn{10}{|l|}{ NLR } \\
\hline$\leq 3$ & 53 & & Ref. & & & & Ref. & & \\
\hline$>3$ & 47 & 0.013 & 1.224 & 1.072 & 1.393 & 0.017 & 1.493 & 1.054 & 2.138 \\
\hline \multicolumn{10}{|c|}{ PA (mg/dl) } \\
\hline$\leq 18$ & 52 & & Ref. & & & & Ref. & & \\
\hline$>18$ & 48 & 0.136 & 1.145 & 0.961 & 1.366 & 0.910 & 1.091 & 0.810 & 1.230 \\
\hline \multicolumn{10}{|c|}{$\mathrm{RBP}(\mathrm{mg} / \mathrm{dl})$} \\
\hline$\leq 2.3$ & 51 & & Ref. & & & & Ref. & & \\
\hline$>2.3$ & 49 & 0.248 & 1.131 & 0.925 & 1.407 & 0.906 & 1.187 & 0.798 & 1.220 \\
\hline \multicolumn{10}{|c|}{$\mathrm{WBC}(/ \mu)$} \\
\hline$\leq 5,960$ & 56 & & Ref. & & & & Ref. & & \\
\hline$>5,960$ & 44 & 0.337 & 0.970 & 0.650 & 1.160 & 0.530 & 1.132 & 0.684 & 1.220 \\
\hline \multicolumn{10}{|c|}{ CRP (mg/ml) } \\
\hline$\leq 1$ & 83 & & Ref. & & & & Ref. & & \\
\hline$>1$ & 17 & 0.739 & 1.050 & 0.970 & 1.130 & 0.814 & 0.996 & 0.866 & 1.030 \\
\hline \multicolumn{10}{|l|}{ SI } \\
\hline$\leq 367$ & 57 & & Ref. & & & & Ref. & & \\
\hline$>367$ & 43 & 0.534 & 1.040 & 0.920 & 1.180 & 0.623 & 0.847 & 0.733 & 1.298 \\
\hline
\end{tabular}

The P-values appearing in variable value name rows represent Wald P-values for the corresponding category of variable. HR, hazard ratio; LB, lower bound; UB, upper bound; Ref., reference category.

response (PR) was significantly higher in the group of patients with a lower NLR than in the high NLR group (38.5 vs. $19.1 \%$, respectively; $\mathrm{P}=0.031$ ) and the percentage of patients with progressive diseases (PD) was higher in the group with a higher NLR (57.4 vs. $25.0 \%$, high vs. low NLR group, respectively; $\mathrm{P}=0.044)$.

NLR and nutrition, immune response and inflammation. Correlations of the NLR with patients' nutritional condition, inflammatory status, and cellular immune responses are shown in Figs. 1-3, respectively. The levels of the NLR were significantly inversely correlated with the serum levels of prealbumin (Fig. 1A, $\mathrm{P}=0.00006$ ) and retinol binding protein (Fig. 1B, $\mathrm{P}=0.00004)$. The NLR levels were also significantly correlated with the levels of CRP (Fig. 2A, $\mathrm{P}=0.00007$ ), white blood cell count (Fig. 2B, $\mathrm{P}=0.00009$ ), and inversely did with SI (Fig. 3, $\mathrm{P}=0.00003$ ).

NLR and prognosis. The relationship between the NLR and survival of stage IV patients with gastric cancer treated with S-1 plus cisplatin is shown in Fig. 4. OS was significantly longer in patients with a lower NLR $(\leq 3.0)$ than in those with a higher NLR (>3.0) $(\mathrm{P}=0.042)$. Range duration of follow-up was 1-1,514 days in the NLR $>3$ arm and 1-1533 days in the NLR $\leq 3$. Median OS was 931 vs. 1,034 days for NLR $>3$ vs. NLR $\leq 3$ [stratified hazard ratio (HR), $0.831 ; 95 \% \mathrm{CI}, 0.513$ to 0.972 ; $\log$ rank $\mathrm{P}=0.042$ ].

Table III shows that patients with a lower NLR $(\leq 3.0)$ was associated with a statistically significant improvement in OS compared with higher NLR (>3.0) (hazard ratio, 1.493; 95\% CI, 1.054-2.138; $\mathrm{P}=0.017)$.

\section{Discussion}

The levels of the NLR were analyzed along with several clinical indicators in patients with Stage IV gastric cancer who were treated with an S-1 plus CDDP regimen as a first-line therapy. The present study has demonstrated that the NLR is a useful marker of malnutrition, systemic inflammation and immune suppression. Moreover, the NLR was demonstrated to be a prognostic indicator in these patients.

It has been reported that various inflammation-related markers such as CRP, the NLR or the GPS are influenced by the production of pro- and anti-inflammatory cytokines and are associated with a poor prognosis in various types of cancer (19-21). In the present study, immune suppression appeared to be closely associated with an increased NLR since the levels of the SI were significantly inversely correlated with an increased NLR. We have previously reported that myeloid-derived suppressor cells (MDSC), which are suggested to increase with cancer or inflammation, are increased, and their circulating levels are related to a decreased SI, malnutrition and increased vascular endothelial growth factor (VEGF) in patients with cancer $(32,33)$. We have speculated that immune suppression may also occur with increased MDSC that are activated by inflammation or with tumor-produced VEGF. 
The reason for the associaton of tumor responses to chemotherapy with the NLR or systemic inflammation. It may be that because malnutrition, immune suppression and PS (performance status) are closely associated with each other in general, patients with a fair PS may have received a higher dose of chemotherapeutic drugs or, alternatively, more chemotherapy, or chemotherapy at a greater rate might have been administered to these patients after 4 cycles of S-1 plus cisplatin.

Another possibility, that of immunogenic cell death (ICD) after chemotherapy or radiotherapy, has been reported (34-36). Moreover, recent studies of immune-checkpoint inhibitors have raised the possibility that lymphocytes of a cancer-bearing host can recognize neoantigens or tumor-specific mutation antigens (37). Since a patient's immune function depends on systemic inflammation, the anti-tumor activity of cytotoxic $\mathrm{T}$ lymphocytes that recognize tumor cells may be suppressed in patients with high inflammation, and the response of these patients to chemotherapy and their prognosis might be poor.

In conclusion, the NLR, which is an inflammatory marker, is considered to be a useful marker for tumor response to chemotherapy, immune suppression, malnutrition and poor prognosis. However, because the NLR is thought to be easily influenced by infection or stress that is not caused by a tumor, ideally an appropriate NLR cut-off level needs to be investigated for its use as a marker in clinical practice. Furthermore, it is possible that the combined use of anti-inflammatory agents with chemotherapeutic agents may be more effective. Further investigations into these points are warranted.

\section{References}

1. Jemal A, Bray F, Center MM, Ferlay J, Ward E and Forman D: Global cancer statistics. CA Cancer J Clin 61: 69-90, 2011.

2. Ferlay J, Shin HR, Bray F, Forman D, Mathers C and Parkin DM: Estimates of worldwide burden of cancer in 2008: GLOBOCAN 2008. Int J Cancer 127: 2893-2917, 2010.

3. Coussens LM and Werb Z: Inflammation and cancer. Nature 420 : 860-867, 2002

4. Balkwill $\mathrm{F}$ and Mantovani A: Inflammation and cancer: Back to Virchow? Lancet 357: 539-545, 2001.

5. Jaiswal M, LaRusso NF, Burgart LJ and Gores GJ: Inflammatory cytokines induce DNA damage and inhibit DNA repair in cholangiocarcinoma cells by a nitric oxide-dependent mechanism. Cancer Res 60: 184-190, 2000.

6. McMilan DC: The systemic inflammation-based Glasgow prognostic score: A decade of experience in patients with cancer. Cancer Treat Rev 39: 534-540, 2013.

7. Smith RA, Bosonnet L, Raraty M, Sutton R, Neoptolemos JP, Campbell F and Ghaneh P: Preoperative platelet-lymphocyte ratio is an independent significant prognostic marker in resected pancreatic ductal adenocarcinoma. Am J Surg 197: 466-472, 2009.

8. Tomita M, Shimizu T, Ayabe T, Yonei A and Onitsuka T: Preoperative neutrophil to lymphocyte ratio as a prognostic predictor after curative resection of non-small lung cancer. Anticancer Res 31: 2995-2998, 2011

9. Pinato DJ, Shiner RJ, Seckl MJ, Stebbing J, Sharma R and Mauri FA: Prognostic performance of inflammation-based prognostic indices in primary operable non-small cell lung cancer. $\mathrm{Br}$ J Cancer 110: 1930-1935, 2014

10. Zhang H, Zhang L, Zhu K, Shi B, Yin Y, Zhu J, Yue D, Zhang B and Wang C: Prognostic significance of combination of preoperative platelet count and neutrophil-lymphocyte ratio (COP-NLR) in patients with non-small cell lung cancer: Based on a large cohort study. PLoS One 10: e0126496, 2015.

11. Shibutani M, Maeda K, Nagahara H, Ohtani H, Iseki Y, Ikeya T, Sugano K and Hirakawa K: The prognostic significance of a postoperative systemic inflammatory response in patients with colorectal cancer. World J Surg Oncol 13: 194, 2015.
12. Albayrak S, Zengin K, Tanik S, Atar M, Unal SH, Imamoglu MA and Gurdal M: Can the neutrophil-to-lymphocyte ratio be used to predict recurrence and progression of non-muscle-invasive bladder cancer? Kaohsiung J Med Sci 32: 327-333, 2016.

13. Dong YW, Shi YQ, He LW and Su PZ: Prognostic significance of neutrophil-to-lymphocyte ratio in rectal cancer: A meta-analysis. Onco Targets Ther 9: 3127-3134, 2016.

14. Nakamura K, Nagasaka T, Nishida T, Haruma T, Ogawa C, Kusumoto T, Seki N and Hiramatsu Y: Neutrophil to lymphocyte ratio in the pre-treatment phase of final-line chemotherapy predicts the outcome of patients with recurrent ovarian cancer. Oncol Lett 11: 3975-3981, 2016.

15. Aino H, Sumie S, Niizeki T, Kuromatsu R, Tajiri N, Nakano M, Satani M, Okamura S, Shimose S, Miyahara K and Torimura T: The systemic inflammatory response as a prognostic factor for advanced hepatocellular carcinoma with extrahepatic metastasis. Mol Clin Oncol 5: 83-88, 2016.

16. Ding PR, An X, Zhang RX, Fang YJ, Li LR, Chen G, Wu XJ, Lu ZH, Lin JZ, Kong LH, et al: Elevated preoperative neutrophil to lymphocyte ratio predicts risk of recurrence following curative resection of stage IIA colon cancer. Int J Colorectal Dis 25: 1427-1433, 2010.

17. Jung MR, Park YK, Jeong O, Seon JW, Ryu SY, Kim DY and Kim YJ: Elevated preoperative neutrophil to lymphocyte ratio predicts poor survival following resection in late stage gastric cancer. J Surg Oncol 104: 504-510, 2011.

18. Stotz M, Gerger A, Eisner F, Szkandera J, Loibner H, Ress AL, Kornprat P, AlZoughbi W, Seggewies FS, Lackner C, et al: Increased neutrophil-lymphocyte ratio is a poor prognostic factor in patients with primary operable and inoperable pancreatic cancer. Br J Cancer 109: 416-421, 2013.

19. Grivennikov SI, Greten FR and Karin M: Immunity, inflammation, and cancer. Cell 140: 883-899, 2010.

20. McMillan DC: Systemic inflammation, nutritional status and survival in patients with cancer. Curr Opin Clin Nutr Metab Care 12: 223-226, 2009.

21. Zahorec R: Ratio of neutrophil to lymphocyte count-rapid and simple parameter of systemic inflammation and stress in critically ill. Bratisl Lek Listy 102: 5-14, 2001 (In English, Slovak).

22. Shirasaka T, Shimamoto Y, Ohshimo H, Yamaguchi M, Kato T, Yonekura K and Fukushima M: Development of a novel form of an oral 5-fluorouracil derivative (S-1) directed to the potentiation of the tumor selective cytotoxicity of 5-fluorouracil by two biochemical modulators. Anticancer Drugs 7: 548-557, 1996.

23. Sugimachi K, Maehara Y, Horikoshi N, Shimada Y, Sakata Y, Mitachi Y and Taguchi T: An early phase II study of oral S-1, a newly developed 5-fluorouracil derivative foe advanced and recurrent gastrointestinal cancers. S-1 Gastrointestinal Cancer Study Group. Oncology 57: 202-210, 1999.

24. Sakata Y, Ohtsu A, Horikoshi N, Sugimachi K, Mitachi Y and Taguchi T: Late phase II study of novel oral fluoropyrimidine anticancer drug S-1 (1 M tegafur- $0.4 \mathrm{M}$ gimestat-1 M otastat potassium) in advanced gastric cancer patients. Eur J Cancer 34: 1715-1720, 1998

25. Koizumi W, Kurihara M, Nakano S and Hasegawa K: Phase II study of S-1, a novel oral derivative of 5-fluorouracil, in advanced gastric cancer. For the S-1 Cooperative Gastric Cancer Study Group. Oncology 58: 191-197, 2000.

26. Japanese Gastric Cancer Association: Japanese gastric cancer treatment guidelines 2010 (ver. 3). Gastric Cancer 14: 113-123, 2011.

27. Shibata M and Gonda K: Cachexia, immunological participation. In: Horizons in Cancer Research vol 58. Nova Science Publishers Inc, New York, 2015.

28. Tisdale MJ: Biology of cachexia. J Natl Cancer Inst 89: 1763-1773, 1997.

29. Shibata M, Nezu T, Kanou H, Abe H, Takekawa M and Fukuzawa M: Decreased production of interleukin-12 and type 2 immune responses are marked in cachectic patients with colorectal and gastric cancer. J Clin Gastroenterol 34: 416-420, 2002.

30. Shibata M, Nagata Y, Kimura T, Kanou H, Nezu T, Takekawa M and Fukuzawa M: Elevated serum concentration of interleukin-1 receptor antagonist (IL-1ra) is correlated to interleukin-6 and to hypoalbuminemia in cachectic patients with colorectal cancer. Int J Clin Oncol 5: 116-120, 2000.

31. Shibata $\mathbf{M}$ and Takekawa M: Increased serum concentration of circulating soluble receptor for interleukin-2 and its effect as a prognostic indicator in cachectic patients with gastric and colorectal cancer. Oncology 56: 54-58, 1999. 
32. Ohki S, Shibata M, Gonda K, Machida T, Shimura T, Nakamura I, Ohtake T, Koyama Y, Suzuki S, Ohto H and Takenoshita S: Circulating myeloid-derived suppressor cells are increased and correlated to immune suppression, inflammation and hypoproteinemia in patients with cancer. Oncol Rep 28: 453-458, 2012.

33. Nakamura I, Shibata M, Gonda K, Yazawa T, Shimura T, Anazaa T, Suzuki S, Sakurai K, Koyama Y, Ohto H, et al: Serum levels of vascular endothelial growth factor are increased and correlated with malnutrition, immunosuppression involving MDSCs and systemic inflammation in patient with cancer of the digestive system. Oncol Lett 5: 1682-1686, 2013.

34. Suzuki Y, Mimura K, Yoshimoto Y, Watanabe M, Ohkubo Y, Izawa S, Murata K, Fujii H, Nakano T and Kono K: Immunogenic tumor cell death induced by chemoradiotherapy in patients with esophageal squamous cell carcinoma. Cancer Res 72: 3967-3976, 2012.
35. Yoshimoto Y, Kono K and Suzuki Y: Anti-tumor immune responses induced by radiotherapy: A review. Fukushima J Med Sci 61: 13-22, 2015.

36. Garg AD, Galluzzi L, Apetoh L, Baert T, Birge RB, Bravo-San Pedro JM, Breckpot K, Brough D, Chaurio R, Cirone M, et al: Molecular and translational classifications of DAMPs in immunogenic cell death. Front Immunol 6: 588, 2015.

37. Wang RF and Wang HY: Immune targets and neoantigens for cancer immunotherapy and precision medicine. Cell Res 27: $11-37,2017$ 